\title{
Light quality regulates flowering in FvFT1/FvTFL1 dependent manner in the woodland strawberry Fragaria
}

\section{vesca}

\section{Marja Rantanen ${ }^{1}$, Takeshi Kurokura ${ }^{1+}$, Katriina Mouhu ${ }^{1}$, Paulo Pinho ${ }^{2}$, Eino Tetri ${ }^{2}$, Liisa Halonen ${ }^{2}$, Pauliina Palonen ${ }^{1}$, Paula Elomaa ${ }^{1}$ and Timo Hytönen ${ }^{1,3 *}$}

${ }^{1}$ Department of Agricultural Sciences, University of Helsinki, Helsinki, Finland

${ }^{2}$ Department of Electrical Engineering and Automation, Aalto University, Espoo, Finland

${ }^{3}$ Department of Biosciences, University of Helsinki, Helsinki, Finland

\section{Edited by:}

George Coupland, Max Planck

Society, Germany

Reviewed by:

Eiji Nambara, University of Toronto,

Canada

Chris Helliwell, CSIRO, Australia

*Correspondence:

Timo Hytönen, Department of

Agricultural Sciences, University of Helsinki, PO Box 27,

Latokartanonkaari 7 ,

Fl-00014 Helsinki, Finland

e-mail: timo.hytonen@helsinki.fi

${ }^{\dagger}$ Present address:

Takeshi Kurokura, Department of Agriculture, Faculty of Agriculture, Utsunomiya University, Tochigi, Japan
Control of flowering in the perennial model, the woodland strawberry (Fragaria vesca L.), involves distinct molecular mechanisms that result in contrasting photoperiodic flowering responses and growth cycles in different accessions. The F. vesca homolog of TERMINAL FLOWER1 (FVTFL1) functions as a key floral repressor that causes short-day (SD) requirement of flowering and seasonal flowering habit in the SD strawberry. In contrast, perpetual flowering $F$. vesca accessions lacking functional FVTFL1 show FLOWERING LOCUS T (FvFT1)-dependent early flowering specifically under long-days (LD). We show here that the end-of-day far-red (FR) and blue (B) light activate the expression of FVFT1 and the F. vesca homolog of SUPPRESSOR OF THE OVEREXPRESSION OF CONSTANS (FVSOC1) in both SD and LD strawberries, whereas low expression levels are detected in red $(\mathrm{R})$ and $\mathrm{SD}$ treatments. By using transgenic lines, we demonstrate that FVFT1 advances flowering under $F R$ and $B$ treatments compared to $R$ and $S D$ treatments in the LD strawberry, and that FVSOC1 is specifically needed for the B light response. In the SD strawberry, flowering responses to these light quality treatments are reversed due to up-regulation of the floral repressor FVTFL 1 in parallel with FvFT1 and FVSOC1. Our data highlights the central role of FVFT1 in the light quality dependent flower induction in the LD strawberry and demonstrates that FVTFL1 reverses not only photoperiodic requirements but also light quality effects on flower induction in the SD strawberry.

Keywords: flowering, Fragaria, FT, light spectrum, perennial, SOC1, strawberry, TFL1

\section{INTRODUCTION}

Plants monitor light intensity, duration, spectrum, and direction to adjust their growth and development. Photoperiod changes regularly throughout the year, and therefore, many plants rely on photoperiodic signals to control important phase transitions including flower induction. Furthermore, specific regions of the light spectrum have different effects on flowering. For example, far-red (FR) light, which is enriched under canopy, causes early flowering in many species (Brown and Klein, 1971; Johnson et al., 1994; Cerdán and Chory, 2003; Wollenberg et al., 2008). Plants sense changes in light by using photoreceptors. Phytochromes (Phy) are the only photoreceptors known to mediate photomorphogenic red (R) and FR signals (Takano et al., 2009; Strasser et al., 2010). In Arabidopsis [Arabidopsis thaliana (L.) Heynh.], PhyA promotes, whereas PhyB, D, and E repress flowering (Johnson et al., 1994; Reed et al., 1994; Devlin et al., 1998, 1999; Hu et al., 2013). In addition, cryptochrome (Cry) and light oxygen voltage (LOV) receptors mediate the effect of blue (B) light to control flowering (Guo et al., 1998; Mockler et al., 1999, 2003; Sawa et al., 2007).
Genes that are regulating photoperiodic flowering are conserved between annual short-day (SD) and long-day (LD) model species rice (Oryza sativa L.) and Arabidopsis, respectively, although their mode of action may differ (Hayama et al., 2003; Tsuji et al., 2011; Brambilla and Fornara, 2013). Photoperiodic flowering is explained by the external coincidence model. In Arabidopsis, CONSTANS (CO) mRNA is expressed rhythmically with a peak around the dusk under LD conditions and during the night under SD (Suarez-Lopez et al., 2001). Since CO is unstable in darkness, the protein accumulates only under LD when light coincides with the $C O$ mRNA expression in the afternoon (Valverde et al., 2004). CO activates FLOWERING LOCUS T (FT) in the leaf phloem, and the FT protein moves into the shoot apical meristem (SAM) (Corbesier et al., 2007; Jaeger and Wigge, 2007; Tamaki et al., 2007). At the SAM, FT binds with the bZIP transcription factor $\mathrm{FD}$, and this complex up-regulates the gene expression of MADS transcription factor APETALA1 (AP1) to induce flowering (Abe et al., 2005; Wigge et al., 2005). Also in SD plant rice, photoperiodic flowering occurs through external coincidence. Rice CO homolog, Heading date 1 (Hd1), has similar expression pattern with $\mathrm{CO}$. However, Hd1 activates FT homolog 
Heading date $3 a(\mathrm{Hd} 3 \mathrm{a})$ only under SD through an unknown mechanism which includes the action of PhyB (Kojima et al., 2002; Cremer and Coupland, 2003; Ishikawa et al., 2011; Tsuji et al., 2011). Furthermore, Hd3a forms a florigen activator complex with 14-3-3 proteins and OsFD1 to activate OsMADS15, a homolog of AP1, to induce flowering (Taoka et al., 2011).

Light quality affects the transcription of CO and FT and the stability of CO protein via different photoreceptors. Yanovsky and Kay (2002) showed that both Cry2 and PhyA are involved in the normal activation of FT mRNA expression in the photoperiodic flowering. These photoreceptors play partially redundant role to mediate $\mathrm{B}$ light promotion of flowering together with Cryl, although the main role of PhyA is to mediate the FR signal (Mockler et al., 2003). These photoreceptors are involved in the stabilization of $\mathrm{CO}$ protein, whereas PhyB promotes the degradation of $\mathrm{CO}$ in $\mathrm{R}$ light (Valverde et al., 2004). B light activated LOV-receptor, FLAVIN BINDING, KELCH REPEAT, F-BOX1 (FKF1), stabilizes CO protein specifically in the afternoon when CO promotes the expression of FT. FKF1 is also directly involved in the transcriptional regulation of both $C O$ and FT (Imaizumi et al., 2003; Sawa et al., 2007; Song et al., 2012). PhyB and other light stable phytochromes have additional roles in shade avoidance conditions. Low $\mathrm{R} / \mathrm{FR}$ ratio inactivates $\mathrm{PhyB}$, which leads to the activation of the photoperiodic flowering pathway. An additional regulator, PHYTOCHROME AND FLOWERING TIME1 (PFT1) promotes the expression of CO and FT by repressing Phy signaling (Cerdán and Chory, 2003; Wollenberg et al., 2008).

In perennials, the molecular level studies on the light regulation of flowering have focused on Populus and on the woodland strawberry, Fragaria vesca L. that represents the model species for the economically important Rosaceae family (Böhlenius et al., 2006; Hsu et al., 2011; Koskela et al., 2012; Mouhu et al., 2013). In F.vesca, both seasonal flowering and perpetual flowering accessions with contrasting photoperiodic responses exist (Brown and Wareing, 1965). Seasonal flowering accessions are SD plants (Heide and Sonsteby, 2007). In perpetual flowering F. vesca, however, LD advances flower induction, but plants eventually flower also under SD (Sønsteby and Heide, 2008; Mouhu et al., 2009; Koskela et al., 2012). A strong floral repressor, F. vesca homolog of TERMINAL FLOWER1 (FvTFL1) has been shown to control seasonal flowering, whereas perpetual flowering accessions have non-functional FvTFL1 alleles with a 2 base pair deletion in the first exon (Iwata et al., 2012; Koskela et al., 2012). Also in the cultivated strawberry (Fragaria $\times$ ananassa Duch.), both seasonal and perpetual flowering cultivars with similar environmental responses are known (Heide, 1977; Sønsteby and Heide, 2007; Bradford et al., 2010; Kurokura et al., 2013).

Molecular analyses in $F$. vesca have revealed that homologs of FT and SUPPRESSOR OF THE OVER-EXPRESSION OF CONSTANS1 (FvFT1 and FvSOC1) may mediate the photoperiodic signal to control flowering through FvTFL1. These genes seem to form a linear pathway in which FvFT1 promotes the expression FvSOC1, which leads to increased FvTFL1 mRNA levels (Mouhu et al., 2013). Since FvTFL1 is a strong floral repressor, the activation of this pathway under LD maintains the plants at the vegetative stage (Koskela et al., 2012). Under SD in autumn, however, the expression of FvFT1 and FvSOC1 decrease leading to the down-regulation of FvTFL1, and consequently, the upregulation of FvAP1 occurs in the shoot apex in parallel with the initiation of floral development. The growth cycle continues in the next spring when determinate inflorescences emerge and produce fruits. Flowering and fruiting overlap with the next yearly growth cycle which begins with the growth of new vegetative axillary shoots with high FvSOC1 and FvTFL1 expression level in the spring. In perpetual flowering accession Hawaii-4 (H4), however, the lack of functional FvTFL1 reverses the photoperiodic flowering response, and both FvFT1 and FvSOC1 act as floral activators (Koskela et al., 2012; Mouhu et al., 2013).

Vince-Prue and Guttridge (1973) showed that the end-of-day FR light treatment prevents flower induction in the cultivated strawberry, whereas R light has an opposite effect. To understand strawberry responses to the light quality at the molecular level, we carried out end-of-day treatments with R, FR and B light in the F. vesca. We report strong activation of FvFT1 by FR light, weaker activation by $\mathrm{B}$ light, and almost no expression under $\mathrm{R}$ light. Using transgenic lines, we show evidence that FvFT1 mediates the promotion of flowering under FR and B light treatments in the perpetual flowering accession $\mathrm{H} 4$, which is lacking functional FvTFL1. In the seasonal flowering accession, however, high FvFT1 expression correlates with high FvTFL1 mRNA levels, and flowering responses to different light qualities are reversed.

\section{MATERIALS AND METHODS PLANT MATERIAL AND GROWING CONDITIONS}

Seedlings of seasonal flowering SD accession of the woodland strawberry (Fragaria vesca L.) and perpetual flowering LD accession H4 (Accession numbers PI551792 and PI551572, respectively; National Clonal Germplasm Repository, Corvallis, USA) were used. Seedlings were raised in a greenhouse under noninductive photoperiod (12 or $18 \mathrm{~h}$ for $\mathrm{H} 4$ and SD F. vesca, respectively) at $18 \pm 1^{\circ} \mathrm{C}$ (first experiment) or at $22 \pm 1^{\circ} \mathrm{C}$. High pressure sodium (HPS) lamps (Airam 400W, Kerava, Finland) were used to supplement natural light with the intensity of $150 \mu \mathrm{mol} \mathrm{m} \mathrm{m}^{-2} \mathrm{~s}^{-1}$. In SD conditions, darkening curtains were used to exclude any light during the $12 \mathrm{~h}$ night. After rooting, seedlings were transplanted to $8 \times 8 \mathrm{~cm}$ pots. Fertilized peat supplemented with $25 \%(\mathrm{v} / \mathrm{v})$ of vermiculite $(\varnothing 2 \mathrm{~mm})$ was used as a growing media. Plants were fertilized with liquid fertilizer biweekly.

Previously reported FvFT1 and FvSOC1 RNAi lines in H4 background (Koskela et al., 2012; Mouhu et al., 2013), and FvFT1 over-expression lines produced in this work (see below), were analyzed. All transgenic plants were propagated from seeds originating from the self-pollination of the primary transgenic lines. Seeds were germinated on moisturized filter paper on petri dishes at room temperature for 5 days when the primary root was emerged. Since both RNAi and over-expression vectors, pK7GWIWG2(II) and p7WG2D (Karimi et al., 2002), contain green fluorescent protein (GFP) as a selectable marker, we observed GFP signal in the primary roots under the fluorescence microscope (Leica MZ FL3, Leice Microsystems, Wetzlar, Germany) and transferred GFP-positive seeds onto the soil. Transgenic seedlings were raised under SD conditions in greenhouse until the light treatments started. FvFT1 
over-expression lines were moved to the light treatments immediately after germination in order to avoid flower induction before the treatments. Wild type control plants were raised following the same procedure with transgenic seedlings.

\section{LIGHTING TREATMENTS}

Incandescent lamps (INC; R/FR =0.95; Philips 60W) and light emitting diodes (LED) were used for the end-of-day lighting treatments. R, FR and B LED lighting systems were built up using deep-red (LZ1-10R205; LEDEngin Inc, San Jose, USA), far-red (L735-66-60; Epitex Inc., Kyoto, Japan) and royal-blue (Z-Power D32282; Seoul Semiconductor Co. Ltd., Ansan-city, Korea) high-power LED components with measured peak wavelength emissions at 655,740 , and $455 \mathrm{~nm}$, respectively. These lighting systems were used in the experiments that did not include transgenic lines. However, in the experiments with transgenic lines, Philips Green Power LED research modules (deep R, FR, and B; Philips, Amsterdam, The Netherlands) were used.

Young seedlings were subjected to the end-of-day light quality treatments in a greenhouse rooms equipped with darkening curtains during the winter season (November-March). The developmental stage of the seedlings in the beginning of the treatments is indicated in the figure legends. Plants were illuminated daily for $12 \mathrm{~h}$ with $150 \mu \mathrm{mol} \mathrm{m} \mathrm{m}^{-2} \mathrm{~s}^{-1}$ of HPS light. After 12-h HPS illumination, the plants were subjected to low intensity $(8-15 \mu \mathrm{mol}$ $\mathrm{m}^{-2} \mathrm{~s}^{-1}$, as indicated in the figure legends) end-of-day R, FR, B or incandescent light (INC) treatments for $6 \mathrm{~h}$. In addition, 12-h SD was used as a control. Natural light was excluded by using darkening curtains when HPS lamps were turned off. Temperature during the treatments was 18 or $22 \pm 1^{\circ} \mathrm{C}$ (indicated in the figure legends). After the treatment period of 5-8 weeks (as indicated in the figure legends), the plants were transferred to standard LD growing conditions, $18 \mathrm{~h}$ of HPS illumination $(150 \mu \mathrm{mol}$ $\mathrm{m}^{-2} \mathrm{~s}^{-1}$ ) at $18 \pm 1^{\circ} \mathrm{C}$, for flowering observations.

\section{GROWTH OBSERVATIONS}

Flowering time observations were carried out 2-3 times per week to record the date of first open flower. In H4, flowering time was also observed by counting the number of leaves in the primary leaf rosette before the terminal inflorescence.

\section{GENETIC TRANSFORMATION}

FvFT1 was amplified by using primers $5^{\prime}$ - aaaagcaggctGGATCA ATATGCCTAGGGACAGG- $3^{\prime}$ and $5^{\prime}$ - agaaagctgggtAAAGGGTT TACGATGATCTTCTC-3' (lower case letters indicate the binding site for the Gateway adapter primers), and the resulting fragment was introduced in the p7WG2D over-expression vector (Karimi et al., 2002), which includes GFP as a selectable marker, using Gateway ${ }^{\circledR}$ technology with Clonase ${ }^{\mathrm{TM}}$ II (Invitrogen, Carlsbad, USA). This construct was electroporated to the Agrobacterium tumefaciens strain GV3101 and transformed to the F.vesca accession H4 as described earlier (Oosumi et al., 2006).

\section{RNA EXTRACTION, cDNA SYNTHESIS, AND REAL-TIME PCR}

Leaf and/or shoot apex samples were collected for gene expression analyses during lighting treatments in the time points indicated in the figures and figure legends. For the leaf samples, middle leaflets of youngest fully opened leaves, and for shoot apex samples, $\sim 1 \mathrm{~mm}$ pieces containing SAM and youngest leaf initials were pooled from several plants. Three biological replicates were collected for each sample. RNA extraction was done by using pine tree method (Monte and Somerville, 2002). For cDNA synthesis (Superscript III reverse transcriptase, Invitrogen) $1 \mu \mathrm{g}$ of total RNA was used. Real time PCR reactions were performed using SYBR Green Master Mix (Roche, Basel, Switzerland) and $3 \mu \mathrm{M}$ primer mix $(\mathrm{F}+\mathrm{R})$ by using LightCycler 480-instrument (Roche). Real time PCR program is presented in Supplementary Figure 1. Three biological and three technical replicates were analyzed in each experiment. Relative expression of selected genes was measured by $\Delta \Delta \mathrm{Ct}$ method with stable FvMSI1 as a normalization gene (Supplementary Figure 2). Real time PCR primers are listed in Supplementary Table1. Primer efficiencies were close to 2 for all primer pairs.

\section{STATISTICAL ANALYSES}

Flowering time results were subjected to the One-Way or TwoWay analysis of variance (ANOVA) using general linear model (GLM-procedure, SAS 9.3 Software, SAS Institute Inc., Cary, USA). Pairwise comparisons were carried out using Tukey's test ( $\alpha=0.05$ or 0.01 in one-way and two-way ANOVA, respectively).

\section{RESULTS}

\section{END-OF-DAY FR, BUT NOT R LIGHT PROMOTES FLOWERING IN THE F.VESCA ACCESSION H4}

Earlier studies in F. vesca have shown that LD advances flowering of the perpetual flowering accession H4 (Mouhu et al., 2009; Koskela et al., 2012). To analyze the effect of light quality on the photoperiodic flower induction in $\mathrm{H} 4$, we subjected seedlings to different end-of-day light quality treatments. Plants with one open leaf were exposed to non-inductive SD ( $12 \mathrm{~h}$ day/ $12 \mathrm{~h}$ night), or SD plus low intensity day extension $(6 \mathrm{~h})$ of FR, R or incandescent light (INC; R:FR ratio of 0.95 ; flower inductive LD control) for 5 weeks at $18^{\circ} \mathrm{C}$ followed by standard LD (18 h) growing conditions (see Materials and methods). In both FR and INC treatments, flower induction was advanced and resulted in terminal inflorescence after about eight leaves in the primary leaf rosette (Figure 1A). In contrast, plants grown under $\mathrm{R}$ treatment flowered after 12 leaves similarly to plants grown under SD.

We further tested the effect of light quality at higher temperature of $22^{\circ} \mathrm{C}$, since earlier study showed that high temperature enhances photoperiodic responses in the perpetual flowering $F$. vesca accessions (Sønsteby and Heide, 2008). Indeed, we found stronger delay of flowering in $\mathrm{R}$ and $\mathrm{SD}$ compared to control plants grown under INC than in the experiment carried out at $18^{\circ} \mathrm{C}$. Plants grown under SD and $\mathrm{R}$ treatments produced $8-9$ leaves more than plants under INC treatment and flowered more than a month later (Supplementary Figure 3). We also tested the effect of B light end-of-day treatment and found that B light advanced flowering in $\mathrm{H} 4$, but the effect was weaker compared to INC light (Supplementary Figure 3).

\section{END-OF-DAY FR LIGHT INDUCES THE EXPRESSION OF THE FVFT1}

Koskela et al. (2012) showed that in H4, FvFT1 expression correlated with floral induction under LD conditions as well as the expression of putative floral meristem identity genes FvAP1 and 


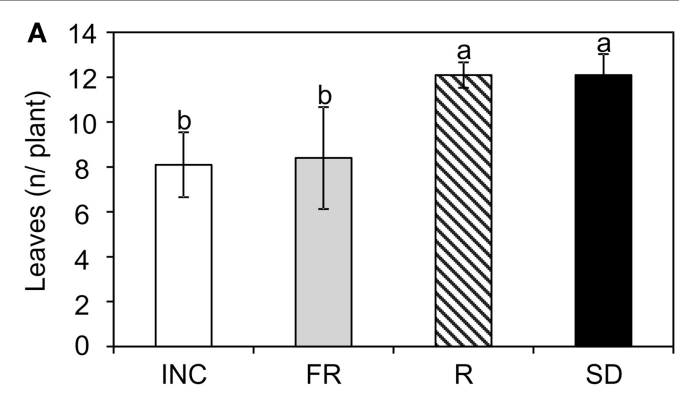

C

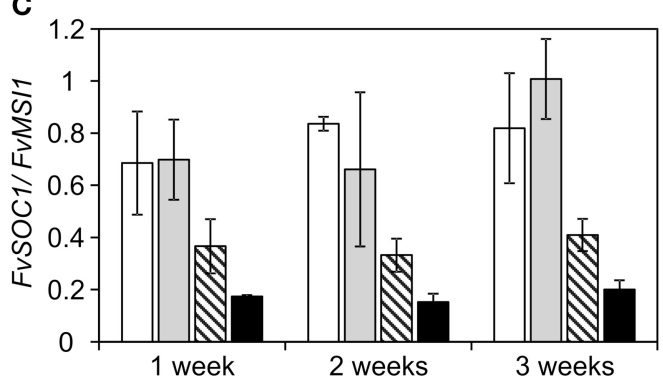

E

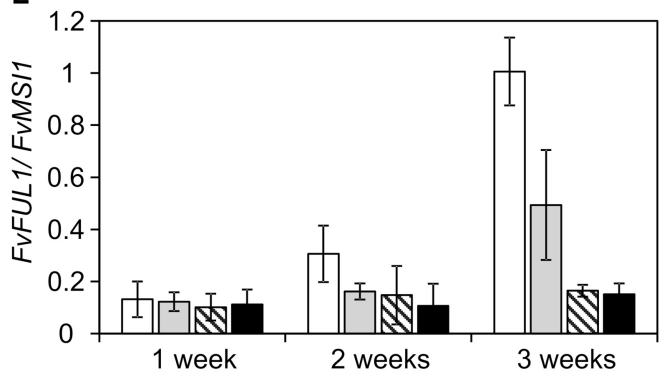

FIGURE 1 | Flowering time and the expression of flowering time genes under the end-of-day light quality treatments in the perpetual flowering F. vesca accession Hawaii-4 (H4). (A) Flowering time of the seedlings of $\mathrm{H} 4$ indicated as the number of leaves in the primary leaf rosette before the terminal inflorescence $(n=8-11)$. Flowering results were subjected to One-Way ANOVA ( $t$-test), $p<0.001$ for the treatment. Different lower-case letters indicate significant differences between the treatments according to Tukey's pairwise test, $\alpha=0.05$. (B) The expression of FVFT1 in the leaves of

FvFUL1. We analyzed the expression of these genes in different end-of-day light quality treatments and found a correlation between the FvFT1 gene expression level and flowering phenotypes. In the leaves of $\mathrm{H} 4, \mathrm{FvFT1}$ was highly expressed in both flowering promoting FR and INC treatments already at 2-leaf stage, 1 week after the beginning of the treatment (Figure 1B). In contrast, we detected very low or no expression in $\mathrm{R}$ and $\mathrm{SD}$ treatments (Figure 1B), in which the plants flowered late.

In Arabidopsis FT activates the expression of SOC1 at the SAM (Moon et al., 2005; Yoo et al., 2005), and this regulatory connection was shown to be conserved in the F. vesca (Mouhu et al., 2013). We found that FvSOC1 mRNA levels in the shoot apices partially correlated with the expression of FvFT1 in the leaves: high expression levels for both genes were detected in INC and FR end-of-day treatments in all tested time points (Figure 1C). However, FvSOC1 mRNA was detected also in R and SD treatments in contrast to FvFT1, but the expression level

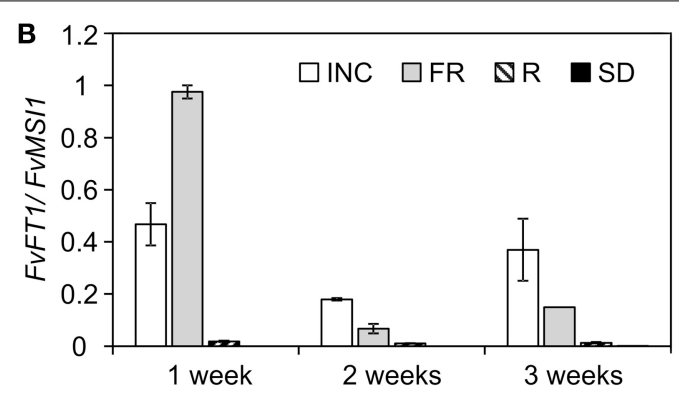

D

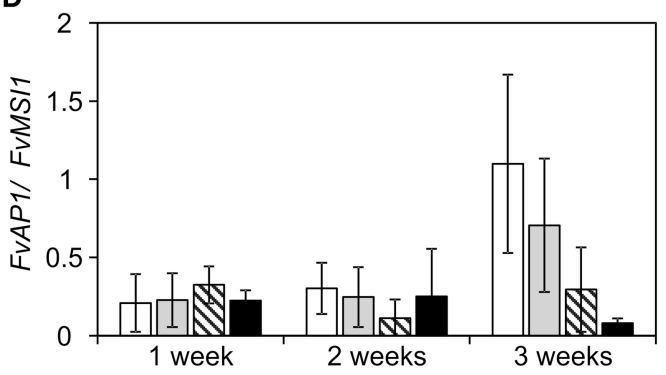

$\mathrm{H} 4$ collected in different time points during the treatments. (C) The expression of FVSOC1, (D) FVAP1, and (E) FVFUL1 in the shoot apex samples of $\mathrm{H} 4$ collected in different time points. Plants with one open leaf were subjected to 12-h short-day (SD) or 12-h SD plus 6-h low intensity $(8 \mu \mathrm{mol}$ $\mathrm{m}^{-2} \mathrm{~s}^{-1}$ ) end-of-day treatment with incandescent, far-red or red light (INC, $F R$, and $\mathrm{R}$, respectively) at $18^{\circ} \mathrm{C}$ for 5 weeks. For gene expression data, three biological replicates were analyzed by real-time PCR. Leaf and shoot apex samples were collected $4 \mathrm{~h}$ after dawn. All results are mean $\pm S D$.

was lower than in FR and INC treatments. The activation of FvFT1 and FvSOC1 in FR and INC treatments was followed by the up-regulation of both FvAP1and FvFUL1 in the shoot apex 3 weeks after the beginning of the treatments but not under SD or $\mathrm{R}$ (Figures 1D,E), indicating that flower induction had occurred only in INC and FR treatments at this stage.

At a higher temperature of $22^{\circ} \mathrm{C}, F v F T 1$ was also strongly upregulated in the leaves of $\mathrm{H} 4$ under FR light compared to $\mathrm{R}$ and SD treatments (Supplementary Figure 3). FvFT1 expression was detected also in B light, but it was several times lower than under FR treatment.

\section{FUNCTIONAL ROLE OF FvFT1 AND FvSOC1 IN LIGHT QUALITY RESPONSES}

To confirm the functional role of FvFT1 in different light quality treatments, we used transgenic approach. First, we overexpressed FvFT1 under cauliflower mosaic virus 35S promoter 
in $\mathrm{H} 4$ background and subjected two independent FvFT1 overexpression lines to different light quality treatments. The ectopic expression of FvFT1 led to extremely early flowering compared to non-transgenic H4 (Figures 2A-D). In addition, FvFT1 overexpression line \#7 showed no differences and line \#5 minor differences in their responses to various end-of-day light quality treatments while in $\mathrm{H} 4$, FR and B light promoted flowering compared to R and SD treatments. Moreover, R light slightly advanced flowering compared to SD in non-transgenic $\mathrm{H} 4$ in this experiment. We also tested the responses of three previously reported FvFT1 RNAi lines in H4 background (Koskela et al., 2012) to the same light quality treatments. In contrast to the wild type H4, FR and $\mathrm{B}$ end-of-day treatments did not advance flowering in two FvFT1 RNAi lines compared to the R and SD treatments, while minor differences between light treatments were observed in the third line (Figure 3A). In this experiment, FR light advanced flowering in non-transgenic $\mathrm{H} 4$ more than B light compared to $\mathrm{SD}$ or $\mathrm{R}$ light treatment, and also $\mathrm{R}$ light slightly promoted flowering compared to SD.

Mouhu et al. (2013) showed that FvSOC1 promotes flowering downstream of FvFT1. In line with this result, silencing of FvFT1 abolished the up-regulation of FvSOC1 and FvFUL1 which was observed in wild type $\mathrm{H} 4$ under FR light treatment (Figures 3B,C). However, B light treatment did not clearly affect the expression of FvSOC1 and FvFUL1 in $\mathrm{H} 4$ at this time point due to differences between the observed flowering times and sampling. In this experiment, the plants that received the B light treatment flowered slightly later than those under FR treatment (Figure 3A).

To understand the role of FvSOC1 in the light quality regulation of flowering, we also studied two independent FvSOC1 RNAi lines in H4 background (Supplementary Figure 4) under the same light quality treatments. Interestingly, FR treatment accelerated flowering of FvSOC1 RNAi plants similarly as in the wild type $\mathrm{H} 4$ while the effect of $\mathrm{B}$ light was absent in the transgenic lines (Figure 3A). This data suggests that FR may induce flowering through FvFT1, independently of FvSOC1, whereas FvSOC1 is needed for early flowering in the end-of-day B light treatment. Taken together, our data on transgenic lines show that FvFT1, in addition to the photoperiodic flowering pathway (Koskela et al., 2012), is the central regulator in the light quality mediated flowering pathway in the perpetual flowering $F$. vesca accession $\mathrm{H} 4$. However, according to our data, FvSOC1 may have more specific role in the $\mathrm{B}$ light regulation of flowering.

\section{FvCO AND FvFT1 EXPRESSION PEAKS DO NOT OVERLAP IN LIGHT QUALITY TREATMENTS}

In Arabidopsis, the expression of $C O$ starts to increase in the afternoon, and $\mathrm{CO}$ protein activates $F T$ expression in late evening specifically under LD (Suarez-Lopez et al., 2001). In addition, light spectrum affects the expression levels of both genes (Imaizumi et al., 2003; Valverde et al., 2004; Kim et al., 2008; Wollenberg et al., 2008). To get insight into to the putative CO/FT module in $F$. vesca, we explored the expression rhythms of $F$. vesca $C O$ and FT homologs in different end-of-day light quality treatments. We focused on daytime expression levels, since our earlier data showed that FvFT1 has a minor expression peak in

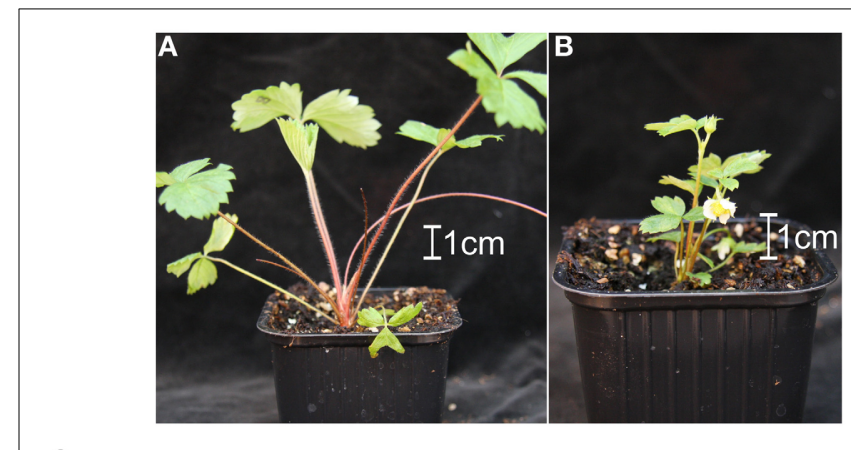

C

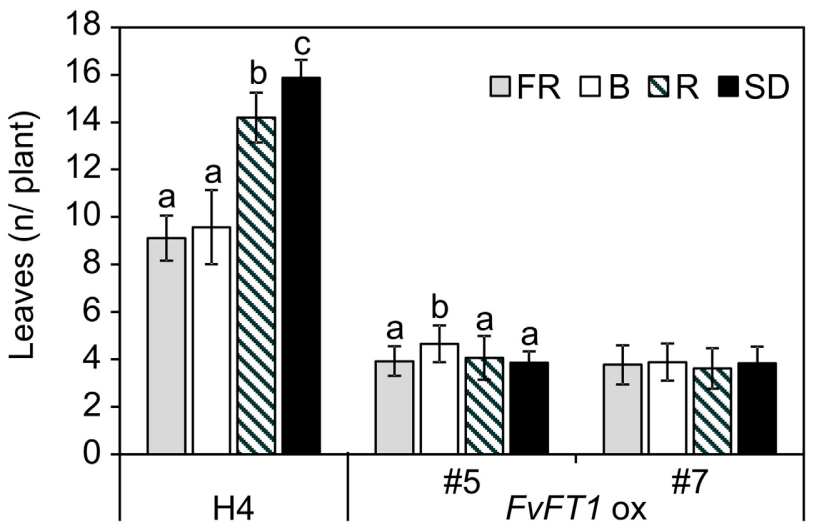

D

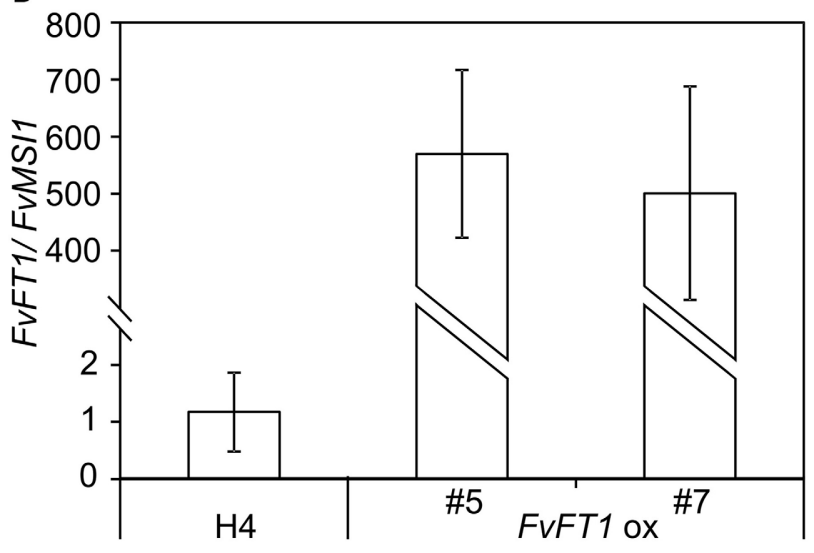

FIGURE 2 | The overexpression of FVFT1 causes extreme early flowering in the perpetual flowering $F$ vesca accession Hawaii-4 (H4). (A) $\mathrm{H} 4$ wild type, and (B) 35S:FvFT1 line \#7 at the age of 8 weeks. (C) Flowering time of FVFT1 overexpression lines in different end-of-day light quality treatments indicated as the number of leaves in the primary leaf rosette before the terminal inflorescence (\#5: $n=21-31, \# 7: n=13-18$, wild type $\mathrm{H} 4: n=36-38)$. Flowering results were subjected to Two-Way ANOVA $(p<0.001$ for each of treatment, genotype, and treatment $\times$ genotype interaction). Pairwise comparisons were carried out for every genotype separately using Tukey's test, $\alpha=0.01$. Treatments with different letters indicate significant differences within the genotype. (D) The expression of FVFT1 in 35S:FVFT1 plants compared to wild type Hawaii-4 under LD (18h). $\mathrm{T}_{1}$ and $\mathrm{H} 4$ seedlings with open cotyledons were subjected to 12-h short-day (SD) or $12-\mathrm{h}$ SD plus 6 -h low intensity $(15 \mu \mathrm{mol}$ $\mathrm{m}^{-2} \mathrm{~s}^{-1}$ ) end-of-day treatment with far-red, red or blue light ( $F R, R$, and $\mathrm{B}$, respectively) at $22^{\circ} \mathrm{C}$ for 8 weeks. For gene expression data, three biological replicates were analyzed by real-time PCR. Leaf samples were collected $16 \mathrm{~h}$ after dawn. All results are mean $\pm S D$. 


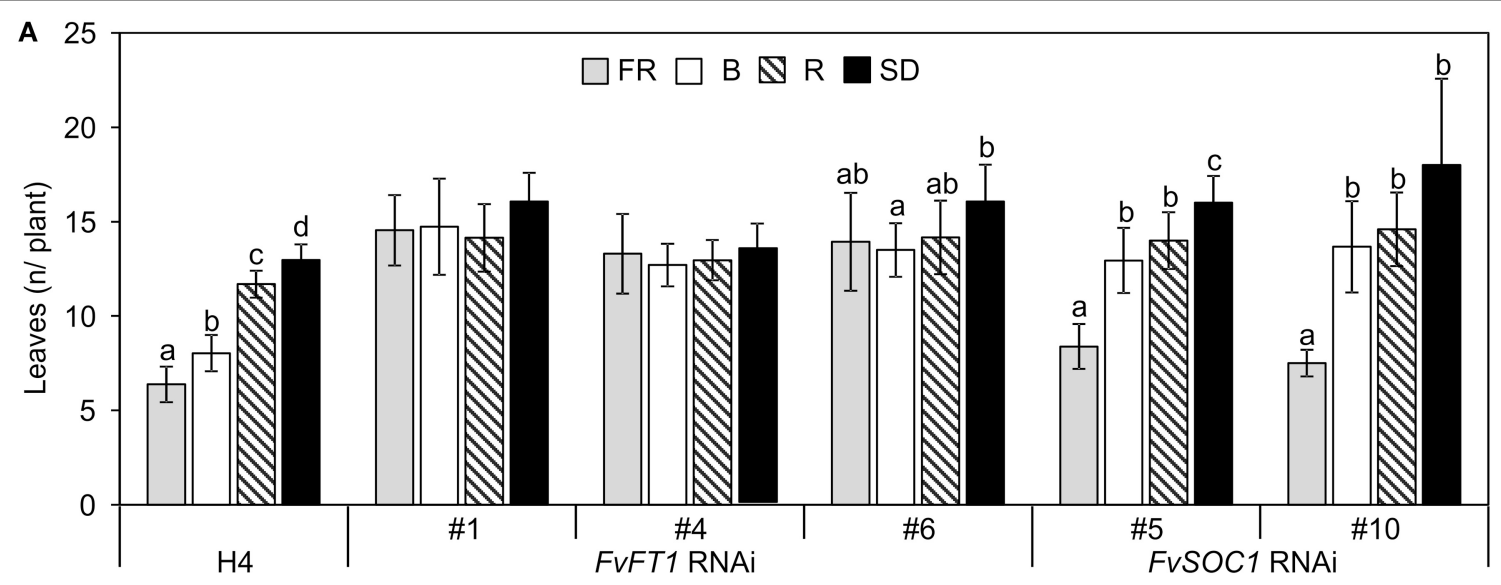

B

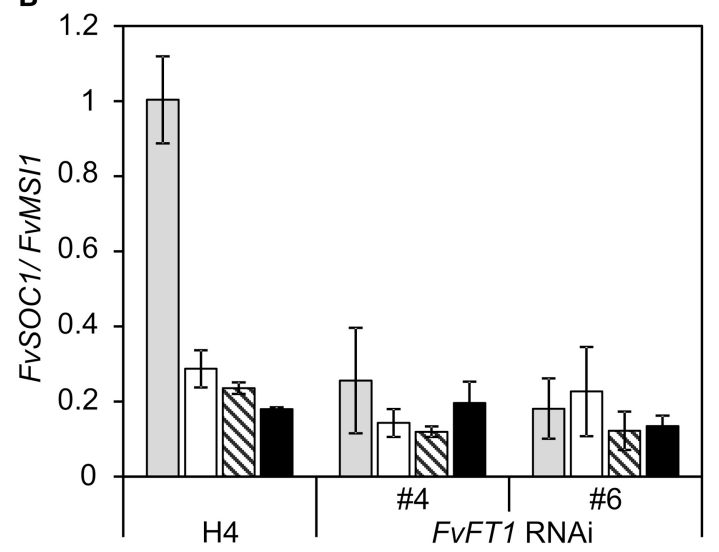

FIGURE 3 | RNAi silencing of FvFT1 and FvSOC1 affects the regulation of flowering by light quality in the perpetual flowering $F$ vesca accession Hawaii-4 (H4). (A) Flowering time of H4, and FvFT1 and FvSOC1 RNAi lines in different end-of-day light quality treatments indicated as the number of leaves in the primary leaf rosette before the terminal inflorescence ( $n=11-23$ for FvFT1 RNAi lines, $n=28-30$ for $\mathrm{H} 4, n=13-18$ for FvSOC1 RNAi-5, $n=5$ for FvSOC1 RNAi-10). Flowering results were subjected to Two-Way ANOVA ( $p<0.001$ for each treatment, genotype, and treatment $\times$ genotype interaction). Pairwise comparisons were carried out for every
C

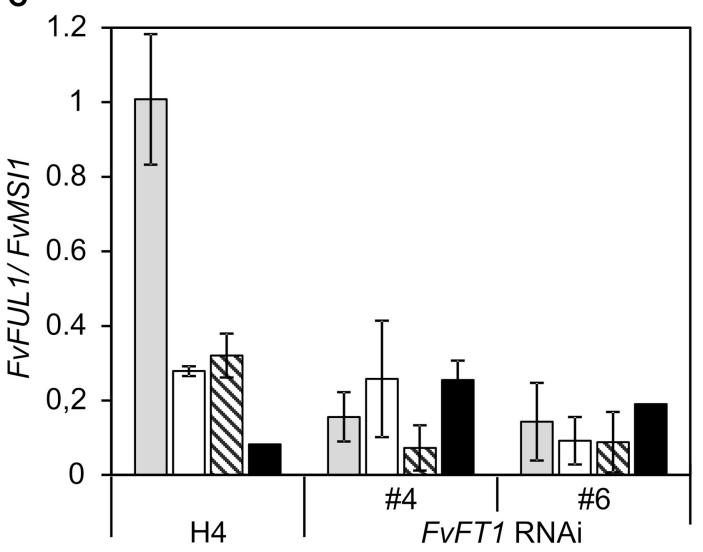

genotype separately using Tukey's test, $\alpha=0.01$. Treatments with different letters indicate significant differences within the genotype. (B) Relative expression of FVSOC1 and (C) FVFUL1 in the shoot apex samples of Hawaii-4 and FVFT1 RNAi lines. $\mathrm{T}_{1}$ and $\mathrm{H} 4$ seedlings with 3-4 open leaves were subjected to 12 -h short-day (SD) or 12-h SD plus 6 -h low intensity $(15 \mu \mathrm{mol}$ $\mathrm{m}^{-2} \mathrm{~s}^{-1}$ ) end-of-day treatment with far-red, red or blue light (FR, $\mathrm{R}$, and $B$, respectively) at $22^{\circ} \mathrm{C}$ for 7 weeks. For gene expression data, three biological replicates were analyzed by real-time PCR. Shoot apex samples were collected $4 \mathrm{~h}$ after dawn. All results are mean $\pm S D$ the morning and another peak in the late evening (Koskela et al., 2012). In the FR treatment, FvFT1 peaked $4 \mathrm{~h}$ after dawn and its expression started to rise again in the evening (Figure 4A). Several times lower expression peaks were detected in B light treatment. Low morning peak ( $4 \mathrm{~h}$ ) was observed also in R light treatment, but the expression level of FvFT1 gene was almost undetectable in the evening.

Several CO homologs have been cloned in the cultivated strawberry, and according to phylogenetic analysis, one of those genes, FrCO, belongs to the group la that includes CO, COL1 and COL2 in Arabidopsis (Griffiths et al., 2003; Stewart, 2007). We searched for F. vesca homologs for $\mathrm{FrCO}$ from the F. vesca genome database (Shulaev et al., 2011; www.rosaceae.org) and found only a single gene with high sequence identity to $\mathrm{FrCO}$ at the nucleotide and protein level (gene04172-v1.0-hybrid; 97 and 96\% identity at the nucleotide and protein level, respectively). We studied the daytime expression rhythm of this gene, which was previously named as FvCO (Shulaev et al., 2011), in different light quality treatments. FvCO peaked at dawn and its expression decreased along the day (Figure 4B; Supplementary Figure 5). Very low expression level was detected in late evening when the mRNA levels of FvFT1 were already rising. In addition, light quality treatments did not affect daytime $F v C O$ gene expression pattern. These data indicates that if the CO-FT connection exists in $F$. vesca, its mode of action differs from Arabidopsis.

\section{END-OF-DAY R LIGHT DOWN-REGULATES FvTFL1 AND INDUCES FLOWERING IN THE SD ACCESSION}

In contrast to the $\mathrm{LD}$ flowering $\mathrm{H} 4$, seasonal flowering SD F. vesca is induced to flower in $12 \mathrm{~h} \mathrm{SD}$, whereas $6 \mathrm{~h}$ day extension with INC treatment after SD prevents flower induction (Koskela et al., 2012). We subjected the SD F. vesca to the similar end-of-day $\mathrm{FR}, \mathrm{R}$ and $\mathrm{B}$ treatments than $\mathrm{H} 4$ and found that its flowering responses to different light qualities were reversed compared to 

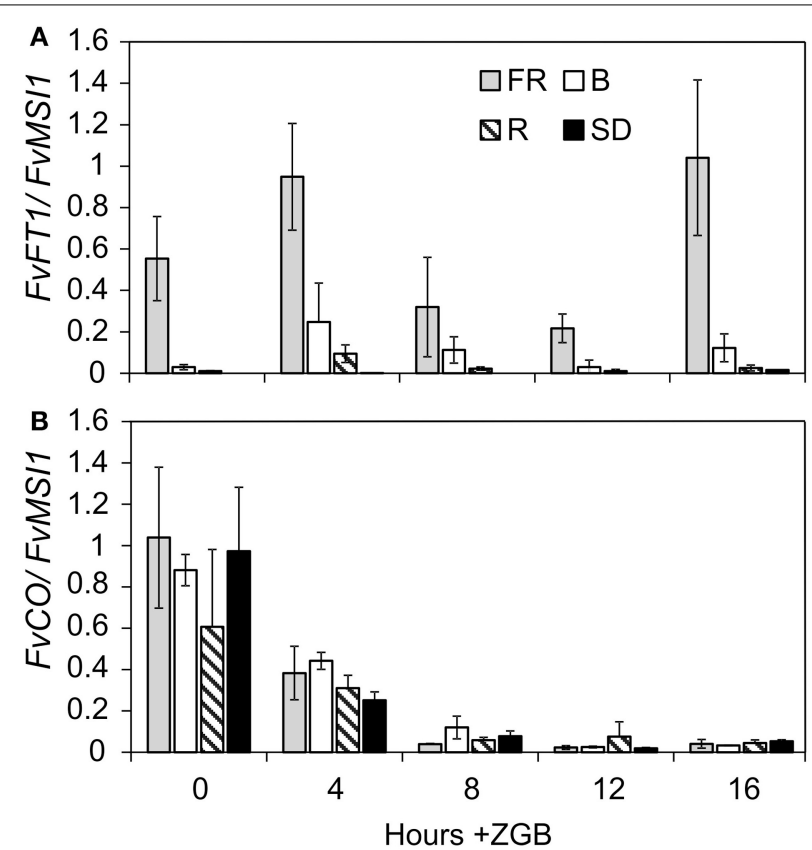

FIGURE 4 | The effect of the end-of-day light quality treatments on the daytime expression of FvFT1 and FvCO in Hawaii-4. (A) Relative

expression of FVFT1 and (B) FvCO in different light quality treatments at 0 , $4,8,12$, and $16 \mathrm{~h}$ after lights were switched on (ZGB = zeitgeber time).

Values are means of three biological replicates $\pm S D$.

H4; $\mathrm{R}$ induced, and FR and B prevented flowering (Figure 5A). In $\mathrm{R}$ and $\mathrm{SD}$ treatments, flowering occurred 38 and 30 days after the end of the 8-week treatment, respectively while the plants under FR and B light treatments stayed vegetative until the end of the experiment.

To understand the observed differences in flowering responses at the molecular level, we analyzed the expression of FvFT1 in the leaves, and FvSOC1 and FvTFL1 mRNA levels in the shoot apex in the SD F. vesca 4 weeks after the beginning of the end-ofday light quality treatments. Similarly to $\mathrm{H} 4$, in the SD F. vesca, FvFT1 was strongly up-regulated by FR light and some expression was detected in B light, whereas in R and SD treatments, the expression was hardly detected or undetectable, respectively (Figure 5B). Like FvFT1 in the leaves, FvSOC1 was up-regulated in the shoot apex of SD F.vesca in FR light and expressed only at low level in both $\mathrm{R}$ and SD treatments, whereas intermediate levels were observed under B light (Figure 5C). The expression of floral repressor, FvTFL1, closely followed that of FvSOC1 in all light quality treatments (Figure 5D). FvTFL1 was several-fold down-regulated in floral-inductive SD and R treatments in comparison to FR and B treatments which inhibited flower induction. Taken together, the effect of end-of-day R, FR or B treatment or SD on the expression of FvFT1 and FvSOC1 was similar in SD F. vesca as in $\mathrm{H} 4$, although the flowering responses were opposite. This difference is associated to FvTFL1 which was co-regulated with FvFT1 and FvSOC1 by light. These data suggest that the functional FvTFL1 reverses flowering response to different light qualities in F.vesca.

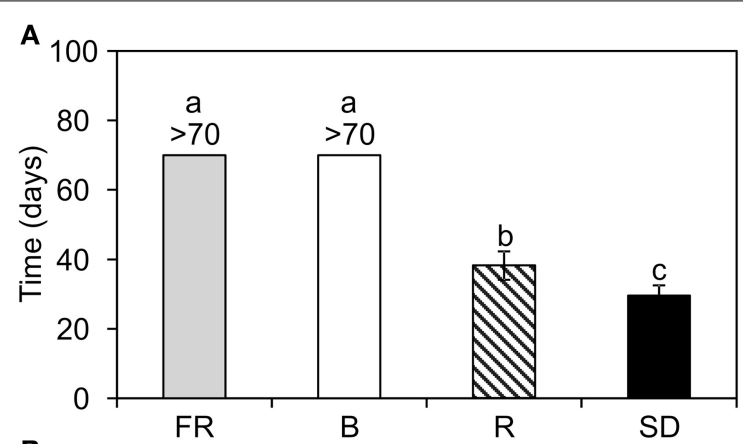

B
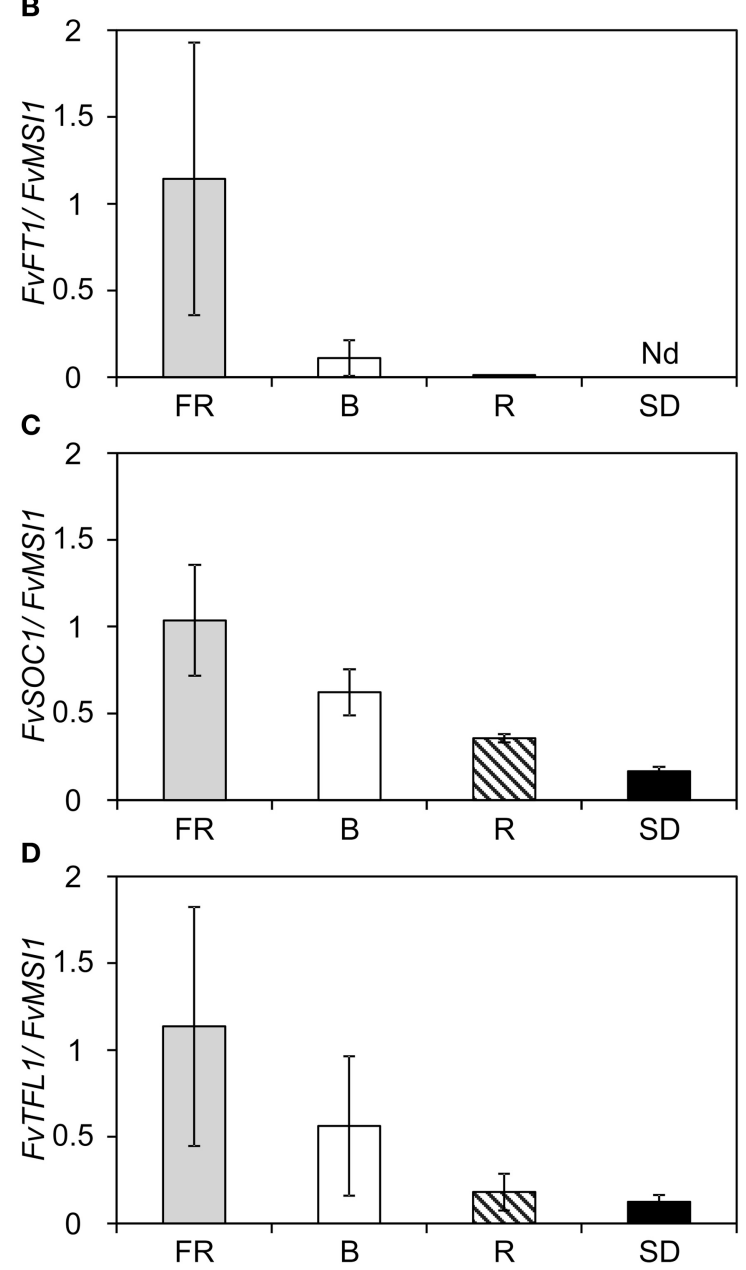

FIGURE 5 | Flowering time and the expression of flowering time genes in the SD $F$ vesca subjected to end-of-day light quality treatments. (A) Flowering time of the SD F. vesca indicated as days to anthesis after the light quality treatments $(n=14)$. Flowering results were subjected to One-Way ANOVA ( $t$-test), $p<0.001$ for the treatment. Different lower-case letters indicate significant differences between the treatments according to Tukey's pairwise test, $\alpha=0.05$. (B) The expression of FVFT1 in the leaf samples, and (C) FVSOC1 and (D) FVTFL1 in the shoot apex samples 4 weeks after the beginning of the light quality treatments. Plants with 5-6 open leaves were subjected to 12-h short-day (SD) or 12-h SD plus 6-h low intensity $\left(15 \mu \mathrm{mol} \mathrm{m} \mathrm{m}^{-2} \mathrm{~s}^{-1}\right)$ end-of-day treatment with blue, far-red or red light ( $\mathrm{B}, \mathrm{FR}$, or $\mathrm{R}$, respectively) at $18^{\circ} \mathrm{C}$ for 8 weeks. For gene expression data, three biological replicates were analyzed by real-time PCR. Shoot apex and leaf samples were collected 4 and $16 \mathrm{~h}$ after dawn, respectively. All results are mean $\pm S D$. $\mathrm{Nd}$, not detected. 


\section{DISCUSSION}

FT has been considered to be a general photoperiodic signaling molecule in both SD and LD plants (Hayama and Coupland, 2004; Lagercrantz, 2009; Pin and Nilsson, 2012). Likewise, in the perpetual flowering $F$. vesca accession $\mathrm{H} 4, \mathrm{FvFT1}$ has recently been reported as an LD-induced floral activator which controls the expression of putative floral meristem identity genes $F v A P 1$ and FvFUL1 (Koskela et al., 2012). Here we show evidence that FvFT1 also mediates the effect of light quality to promote flowering in H4. However, in the seasonal flowering SD F.vesca with a functional FvTFL1, the effects of the light spectra on flowering are reversed.

\section{END-OF-DAY FR AND B LIGHT PROMOTE FLOWERING IN H4}

Flowering of $\mathrm{H} 4$ was advanced by the end-of-day treatment of FR or FR-rich incandescent light whereas R light had no effect or very weak effect compared to the SD control. This is a typical response of various LD plants to light quality (Meijer, 1959; Brown and Klein, 1971; Holland and Vince, 1971; Downs and Thomas, 1982; Martinez-Zapater and Somerville, 1990). Since phytochromes are the sole R/FR receptors mediating photomorphogenic and photoperiodic responses (Takano et al., 2009; Strasser et al., 2010), we suggest that these photoreceptors have a major role in the control flowering also in strawberries. However, further studies are needed to confirm which phytochrome(s) mediate the R/FR responses observed in $\mathrm{H} 4$.

B light has also been shown to promote flowering in Arabidopsis (Bagnall, 1996; Mockler et al., 2003). We found that the end-of-day B light treatment promoted flowering in H4. However, the effect of B light on flowering was weaker than the effect of the FR or INC light in two out of three experiments reported here. Although these results further suggest that phytochromes are major photoreceptors regulating flowering in $F$. vesca, also B light receptor(s) likely have a role in the control of flowering. In Arabidopsis, cryptochromes and the LOV receptor FKF1 are involved in the B light regulation of flowering (Guo et al., 1998; Imaizumi et al., 2003; Valverde et al., 2004). PhyA, however, can also absorb B light, and it mediates B light signal to control flowering at least in the crylcry2 double mutant (Mockler et al., 2003).

\section{FvFT1 AND FVSOC1 HAVE DISTINCT ROLES IN THE LIGHT QUALITY REGULATION OF FLOWERING}

In Arabidopsis, light signals mediated by different photoreceptors control the expression of $F T$ that promotes flowering (Imaizumi et al., 2003; Mockler et al., 2003; Valverde et al., 2004; Song et al., 2012). Consistent with the up-regulation of FT by FR light or low R/FR ratio (Cerdán and Chory, 2003; Mockler et al., 2003; Wollenberg et al., 2008), we found that the end-of-day FR and INC light with R/FR ratio of 0.95 strongly up-regulated FvFT1 in the leaves of H4, whereas FvFT1 expression level was very low under R and SD treatments. Also the end-of-day B light treatment somewhat up-regulated FvFT1 compared to SD. However, consistent with later flowering under B light compared to FR light, several times lower FvFT1 expression level was detected under $\mathrm{B}$ light treatment. The analysis of transgenic lines confirmed the role of FvFT1 in the light quality regulation of flowering in H4.
The overexpression of FvFT1 in H4 caused extreme early flowering independently of the end-of-day light treatment, whereas RNAi silencing of FvFT1 abolished the FR and B light promotion of flowering. These results indicate that FvFT1 does not only activate flowering under LD (Koskela et al., 2012) but also controls flowering according to light quality signals perceived by phytochromes and B light receptors.

FT is a positive regulator of SOC1 in Arabidopsis (Yoo et al., 2005; Torti et al., 2012), and this regulatory link is present also in the F.vesca (Mouhu et al., 2013). In this study, we found highest FvSOC1 expression levels in FR treatment, where the FvFT1 expression level was also highest. Furthermore, the silencing of FvFT1 prevented the up-regulation of $F v S O C 1$ by FR light indicating that FvFT1 mediates at least the FR light regulation of FvSOC1. However, FvFT1 can control flowering independently of FvSOC1 under FR light, since the silencing of FvSOC1 did not affect flowering time under FR light. This is in line with the observation that $F T$ and $S O C 1$ act redundantly to promote flowering under FR enriched light in Arabidopsis (Kim et al., 2008). Although the end-of-day B light treatment advanced flowering compared to SD in non-transgenic H4, this did not occur in FvSOC1 RNAi lines suggesting that FvSOC1 is needed specifically for the B light promotion of flowering. Taken together, both FvFT1 and FvSOC1 are involved in the control of flowering by the end-of-day B treatment, whereas the promotion of flowering by the FR treatment can occur independently of FvSOC1.

\section{FUNCTIONAL FvTFL1 REVERSES THE END-OF-DAY LIGHT QUALITY RESPONSES IN F. VESCA}

Several lines of data support the presence of FvFT1-FvSOC1FvTFL1 regulatory pathway in the SD F. vesca. FvFT1 up-regulates FvSOC1 in the shoot apex at least in H4, and FvSOC1 activates the expression of FvTFL1 that encodes a strong floral repressor. Therefore, the photoperiodic flowering response is reversed in the SD F. vesca compared to H4 (Koskela et al., 2012; Mouhu et al., 2013). We found that in the SD F. vesca, similarly to H4, $F v F T 1$ and $F v S O C 1$ gene expression levels were higher under the end-of-day FR and B light treatments compared to $\mathrm{R}$ and SD treatments. However, in contrast to $\mathrm{H} 4$, SD F. vesca was induced to flower under R light and SD treatments while FR and B light inhibited flowering. Taken together, the expression of FvFT1 and FvSOC1 correlated negatively with the flower induction in the SD F. vesca in all light quality treatments tested in this study as well as in the photoperiodic treatments in previous works (Figure 5; Koskela et al., 2012; Mouhu et al., 2013). We also found that the expression of FvTFL1 closely followed that of $F v S O C 1$ in all light quality treatments indicating that the presence of the functional FvTFL1 not only reverses photoperiodic flowering response (Koskela et al., 2012), but also the effect of the end-of-day B light and the phytochrome-mediated R/FR light on flowering. The ortholog of FvTFL1 may control light responses also in the cultivated strawberry, since the SD cultivar of the cultivated strawberry responds to R/FR treatments similarly to the SD F. vesca (Vince-Prue and Guttridge, 1973) and the F. vesca is one of its ancestors (Hirakawa et al., 2013). Although significant amount of data support the presence of FvFT1-FvSOC1-FvTFL1 pathway in the SD F. vesca, functional analysis is needed to 
confirm whether FvFT1 acts as an anti-florigen in the presence of FvTFL1. Antiflorigens have recently been reported in sugar beet and chrysanthemum (Pin et al., 2010; Higuchi et al., 2013).

\section{FvCO GENE EXPRESSION DO NOT COINCIDE WITH FvFT1 mRNA PEAK}

In the $F$. vesca, FvFT1 mRNA expression peaks in the late evening under LD (Koskela et al., 2012), similarly to FT homologs in several other species (Cremer and Coupland, 2003; Böhlenius et al., 2006; Pin et al., 2010). In Arabidopsis, FT is induced by CO when $C O$ mRNA expression peak coincide with light in the evening under LD (Suarez-Lopez et al., 2001). Our data do not support similar regulation in the F. vesca. The Arabidopsis coincidence model would require $F v C O$ to peak in the afternoon before FvFT1. However, FvFT1 is highly up-regulated $16 \mathrm{~h}$ after dawn, when the $F v C O$ expression level is low, and FvCO mRNA level peaks later toward dawn similarly to Arabidopsis COL2 and Populus deltoides CO1 and CO2, which have little or no effect on the onset of flowering (Ledger et al., 2001; Hsu et al., 2012).

Both FR and B light increase the expression of CO and FT in Arabidopsis, whereas lower mRNA levels are observed in $\mathrm{R}$ rich light (Imaizumi et al., 2003; Valverde et al., 2004; Kim et al., 2008; Wollenberg et al., 2008). Although light quality treatments strongly affected FvFT1 mRNA levels in F. vesca and caused an additional FvFT1 expression peak $4 \mathrm{~h}$ after dawn, the end-of-day light quality treatments had no effect on FvCO daytime expression. Since light quality affects the stability of CO protein in Arabidopsis (Valverde et al., 2004), one possible scenario is that the stabilization of FvCO in the morning up-regulates FvFT1. However, further studies are needed to reveal whether FvCO has a role in the regulation of FvFT1 in different phases of the diurnal cycle in the F. vesca.

\section{CONCLUDING REMARKS}

We have shown that both B and R/FR light signals contribute to the regulation of flowering and flowering time genes in F. vesca, although phytochrome mediated R/FR signals have stronger effect at least in the end-of-day treatments (Figure 6). In the perpetual flowering LD accession $\mathrm{H} 4$, the floral promoter FvFT1 has a central role in flowering responses to different light qualities, whereas FvSOC1 seems to be specifically required for B light mediated activation of flowering. In the SD F. vesca, however, the flowering response to different light spectra is reversed, because of the up-regulation of the strong floral repressor FvTFL1 by FvFT1/FvSOC1. These new insights highlight the importance of the regulation of FvTFL1 and FvFT1 also in the light quality responses, in addition to photoperiodic flowering, in the perennial Rosaceae model plant F. vesca (Koskela et al., 2012). Our result, that flowering of both perpetual (LD) and seasonal (SD) flowering strawberries can be controlled by light quality treatments, may have practical applications in the strawberry cultivation under controlled climate.

\section{AUTHOR CONTRIBUTIONS}

Marja Rantanen, Pauliina Palonen, Paula Elomaa and Timo Hytönen planned the study. Marja Rantanen, Takeshi Kurokura and Katriina Mouhu carried out the experiments and analyzed the data. Paulo Pinho, Eino Tetri and Liisa Halonen designed and

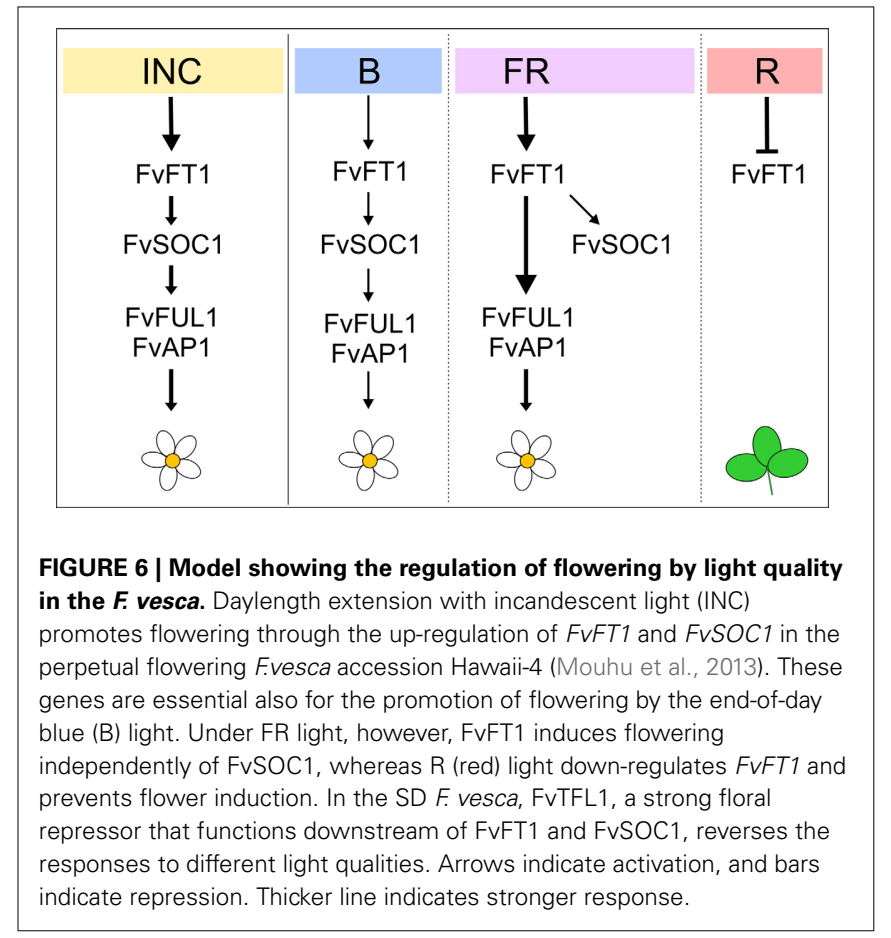

Paulo Pinho constructed the LED luminaires. Marja Rantanen and Timo Hytönen wrote the manuscript with input from other authors. All authors read and approved the final manuscript.

\section{ACKNOWLEDGMENTS}

The work was funded by Academy of Finland (Grant 122946 to Eino Tetri and Pauliina Palonen) and University of Helsinki (Grant DW-4881545211 to Timo Hytönen). Marja Rantanen was supported by grants from the Jenny and Antti Wihuri Foundation and the Aino and August Johannes Tiura Foundation. Marja Rantanen and Katriina Mouhu belong to the Finnish Doctoral Program in Plant Science.

\section{SUPPLEMENTARY MATERIAL}

The Supplementary Material for this article can be found online at: http://www.frontiersin.org/journal/10.3389/fpls.2014.00271/ abstract

\section{Supplementary Table 1 | qPCR primers used in this study.}

Supplementary Figure 1 | qPCR program used in this study.

Supplementary Figure 2 | Stability of the normalization gene FvMSI1.

Supplementary Figure 3 | Flowering time and FvFT1 expression in Hawaii-4 at $22^{\circ} \mathrm{C}$.

Supplementary Figure 4 | The down-regulation of FvSOC1 in the RNAi-lines.

Supplementary Figure 5 | Diurnal expression rhythm of $F v C O$ in the SD $F$ vesca and Hawaii-4.

\section{REFERENCES}

Abe, M., Kobayashi, Y., Yamamoto, S., Daimon, Y., Yamaguchi, A., Ikeda, Y., et al. (2005). FD, a bZIP protein mediating signals from the floral pathway integrator FT at the shoot apex. Science 309, 1052-1056. doi: 10.1126/science.1115983

Bagnall, D. J. (1996). Blue-light promotion of flowering is absent in hy4 mutants of Arabidopsis. Planta 200, 278-280. 
Böhlenius, H., Huang, T., Charbonnel-Campaa, L., Brunner, A. M., Jansson, S., Strauss, S. H., et al. (2006). CO/FT regulatory module controls timing of flowering and seasonal growth cessation in trees. Science 312, 1040-1043. doi: $10.1126 /$ science. 1126038

Bradford, E., Hancock, J. F., and Warner, R. M. (2010). Interactions of temperature and photoperiod determine expression of repeat flowering in strawberry. J. Amer. Soc. Hort. Sci. 135, 102-107.

Brambilla, V., and Fornara, F. (2013). Molecular control of flowering in response to day length in rice. J. Integr. Plant Biol. 55, 410-418. doi: 10.1111/jipb.12033

Brown, J. A. M., and Klein, W. H. (1971). Photomorphogenesis in Arabidopsis thaliana (L) Heynh - threshold intensities and blue-far-red synergism in floral induction. Plant Physiol. 47, 393-399. doi: 10.1104/pp.47.3.393

Brown, T., and Wareing, P. F. (1965). The genetical control of the everbearing habit and three other characters in varieties of Fragaria vesca. Euphytica 14, 97-112.

Cerdán, P. D., and Chory J. (2003). Regulation of flowering time by light quality. Nature 423, 881-885. doi:10.1038/nature01636

Corbesier, L., Vincent, C., Jang, S., Fornara, F., Fan, Q., Searle, I., et al. (2007). FT protein movement contributes to long-distance signaling in floral induction of Arabidopsis. Science 316, 1030-1033. doi: 10.1126/science. 1141752

Cremer, F., and Coupland, G. (2003). Distinct photoperiodic responses are conferred by the same genetic pathway in Arabidopsis and in rice. Trends Plant Sci. 8, 405-407. doi: 10.1016/S1360-1385(03)00192-4

Devlin, P., Patel, S. R., and Whitelam, G. C. (1998). Phytochrome E influences internode elongation and flowering time in Arabidopsis. Plant Cell 10, 1479-1487. doi: 10.1105/tpc.10.9.1479

Devlin, P., Robson, P. R. H., Patel, S. R., Goosey, L., Sharrock, R. A., and Whitelam, G. C. (1999). Phytochrome D acts in the shade-avoidance syndrome in Arabidopsis by controlling elongation growth and flowering time. Plant Physiol. 119, 909-915. doi:10.1104/pp.119.3.909

Downs, R., and Thomas, J. (1982). Phytochrome regulation of flowering in the long-day plant, Hyoscyamus niger. Plant Physiol. 70, 898-900. doi: 10.1104/pp.70.3.898

Griffiths, S., Dunford, R. P., Coupland, G., and Laurie, D. A. (2003). The evolution of CONSTANS-like gene families in barley, rice, and Arabidopsis. Plant Physiol. 131, 1855-1867. doi: 10.1104/pp.102.016188

Guo, H., Yang, H., Mockler, T. C., and Lin, C. (1998). Regulation of flowering time by Arabidopsis photoreceptors. Science 279, 1360-1363. doi: 10.1126/science. 279.5355 .1360

Hayama, R., and Coupland, G. (2004). The molecular basis of diversity in the photoperiodic flowering responses of Arabidopsis and rice. Plant Physiol. 135, 2677-2684. doi: 10.1104/pp.104.042614

Hayama, R., Yokoi, S., Tamaki, S., Yano, M., and Shimamoto K. (2003). Adaptation of photoperiodic control pathways produces short-day flowering in rice. Nature 422, 719-722. doi: 10.1038/nature01549

Heide, O. M. (1977). Photoperiod and temperature interactions in growth and flowering of strawberry. Physiol. Plantarum 40, 21-26. doi: 10.1111/j.13993054.1977.tb01486.x

Heide, O. M., and Sonsteby, A. (2007). Interactions of temperature and photoperiod in the control of flowering of latitudinal and altitudinal populations of wild strawberry (Fragaria vesca). Physiol. Plant. 130, 280-289. doi: 10.1111/j.13993054.2007.00906.x

Higuchi, Y., Narumi, T., Oda, A., Nakano, Y., Sumimoto, K., Fukai, S., et al. (2013). The gated induction system of a systemic floral inhibitor, antiflorigen, determines obligate short-day flowering in chrysanthemums. Proc. Natl. Acad. Sci. U.S.A. 110, 17137-17142. doi: 10.1073/pnas.1307617110

Hirakawa, H., Shirasawa, K., Kosugi, S., Tashiro, K., Nakayama, S., Yamada, M., et al. (2013). Dissection of the octoploid strawberry genome by deep sequencing of the genomes of Fragaria species. DNA Res. 21, 169-181. doi: 10.1093/dnares/dst049

Holland, R. W. K., and Vince, D. (1971). Floral initiation in Lolium temulentum L.: the role of phytochrome in responses to red and far-red light. Planta 98, 232-243.

Hsu, C., Adams, J. P., Kim, H., No, K., Ma, C., Strauss, S. H., et al. (2011). FLOWERING LOCUS T duplication coordinates reproductive and vegetative growth in perennial poplar. Proc. Natl. Acad. Sci. U.S.A. 108, 10756-10761. doi: 10.1073/pnas.1104713108

Hsu, C., Adams, J. P., No, K., Liang, H., Meilan, R., Pechanova, O., et al. (2012). Overexpression of constans homologs $\mathrm{CO} 1$ and $\mathrm{CO} 2$ fails to alter normal reproductive onset and fall bud set in woody perennial poplar. PLoS ONE 7:e45448. doi: 10.1371/journal.pone.0045448

Hu, W., Franklin, K. A., Sharrock, R. A., Jones, M. A., Harmer, S. L., and Lagarias, J. C. (2013). Unanticipated regulatory roles for Arabidopsis phytochromes revealed by null mutant analysis. Proc. Natl. Acad. Sci. U.S.A. 110, 1542-1547. doi: $10.1073 /$ pnas. 1221738110

Imaizumi, T., Tran, H., Swartz, T., Briggs, W., and Kay, S. (2003). FKF1 is essential for photoperiodic-specific light signalling in Arabidopsis. Nature 426, 302-306. doi: $10.1038 /$ nature 02090

Ishikawa, R., Aoki, M., Kurotani, K., Yokoi, S., Shinomura T., Takano, M., et al. (2011). Phytochrome B regulates Heading date 1 (Hdl)-mediated expression of rice florigen $H d 3 a$ and critical day length in rice. Mol. Genet. Genomics 285, 461-470. doi: 10.1007/s00438-011-0621-4

Iwata, H., Gaston, A., Remay, A., Thouroude, T., Jeauffre, J., Kawamura, K., et al. (2012). The TFL1 homologue KSN is a regulator of continuous flowering in rose and strawberry. Plant J. 69, 116-125. doi: 10.1111/j.1365-313X.2011.04776.x

Jaeger, K. E., and Wigge, P. A. (2007). FT protein acts as a longrange signal in Arabidopsis. Curr. Opin. Plant Biol. 17, 1050-1054. doi: 10.1016/j.cub.2007.05.008

Johnson, E., Bradley, M., Harberd, N. P., and Whitelam, G. C. (1994). Photoresponses of light-grown phyA mutants of Arabidopsis. Plant Physiol. 105, 141-149. doi: 10.1104/pp.105.1.141

Karimi, M., Inzé, D., and Depicker, A. (2002). GATEWAY vectors for Agrobacterium -mediated plant transformation. Trends Plant Sci. 7, 193-195. doi: 10.1016/S1360-1385(02)02251-3

Kim, S. Y., Yu, X., and Michaels, S. D. (2008). Regulation of CONSTANS and FLOWERING LOCUS T expression in response to changing light quality. Plant Physiol. 148, 269-279. doi: 10.1104/pp.108.122606

Kojima, S., Takahashi, Y., Kobayashi, Y., Monna, L., Sasaki T., Araki T., et al. (2002). $H d 3 a$, a rice ortholog of the Arabidopsis FT gene, promotes transition to flowering downstream of $\mathrm{Hdl}$ under short-day conditions. Plant Cell Physiol. 43, 1096-1105. doi: 10.1093/pcp/pcf156

Koskela, E. A., Mouhu, K., Albani, M. C., Kurokura, T., Rantanen, M., Sargent, D. J., et al. (2012). Mutation in TERMINAL FLOWER1 reverses the photoperiodic requirement for flowering in the wild strawberry Fragaria vesca. Plant Physiol. 159, 1043-1054. doi: 10.1104/pp.112.196659

Kurokura, T., Mimida, N., Battey, N. H., and Hytönen, T. (2013). The regulation of seasonal flowering in the Rosaceae. J. Exp. Bot. 64, 4131-4141. doi: $10.1093 /$ jxb/ert 233

Lagercrantz, U. (2009). At the end of the day: a common molecular mechanism for photoperiod responses in plants? J. Exp. Bot. 60, 2501-2515. doi: 10.1093/jxb/erp139

Ledger, S., Strayer, C., Ashton, F., Kay, S. A., and Putterill, J. (2001). Analysis of the function of two circadian-regulated CONSTANS-LIKE genes. Plant J. 26, 15-22. doi: 10.1046/j.1365-313x.2001.01003.x

Martinez-Zapater, J. M., and Somerville, C. R. (1990). Effect of light quality and vernalization on late-flowering mutants of Arabidopsis thaliana. Plant Physiol. 92, 770-776. doi: 10.1104/pp.92.3.770

Meijer, G. (1959). "Photomorphogenesis in different spectral regions," in Photoperiodism and Related Phenomena in Plants and Animals, ed R. B. Withrow (Washington, DC: American association for the advancement of science), 101-109.

Mockler, T., Yang, H., Yu, X., Parikh, D., Cheng, Y., Dolan, S., et al. (2003). Regulation of photoperiodic flowering by Arabidopsis photoreceptors. Proc. Natl. Acad. Sci. U.S.A. 100, 2140-2145. doi: 10.1073/pnas.0437826100

Mockler, T. C., Guo, H. W., Yang, H. Y., Duong, H., and Lin, C. T. (1999). Antagonistic actions of Arabidopsis cryptochromes and phytochrome B in the regulation of floral induction. Development 126, 2073-2082.

Monte, D., and Somerville, S. (2002). "Pine tree method for isolation of plant RNA," in DNA Microarrays: A Molecular Cloning Manual, eds D. Bowtel and J. Sambrook (New York, NY: Cold Spring Harbour Laboratory Press), 124-126.

Moon, J., Lee, H., Kim, M., and Lee, I. (2005). Analysis of flowering pathway integrators in Arabidopsis. Plant Cell Physiol. 46, 292-299. doi: 10.1093/pcp/pci024

Mouhu, K., Hytonen, T., Folta, K., Rantanen, M., Paulin, L., Auvinen, P., et al. (2009). Identification of flowering genes in strawberry, a perennial SD plant. BMC Plant Biol. 99:122. doi: 10.1186/1471-2229-9-122

Mouhu, K., Kurokura, T., Koskela, E. A., Albert, V. A., Elomaa, P., and Hytonen, T. (2013). The Fragaria vesca homolog of SUPPRESSOR OF OVEREXPRESSION 
OF CONSTANS1 represses flowering and promotes vegetative growth. Plant Cell 25, 3296-3310. doi: 10.1105/tpc.113.115055

Oosumi, T., Hope, A., Gruszewski, H. A., Blischak, L. A., Baxter, A. J., Wadl, P. A., et al. (2006). High-efficiency transformation of the diploid strawberry (Fragaria vesca) for functional genomics. Planta 223, 1219-1230. doi: 10.1007/s00425005-0170-3

Pin, P. A., Benlloch, R., Bonnet, D., Wremerth-Weich, E., Kraft, T., Gielen, J. J. L., et al. (2010). An antagonistic pair of FT homologs mediates the control of flowering time in sugar beet. Science 330, 1397-1400. doi: 10.1126/science.1197004

Pin, P. A., and Nilsson, O. (2012). The multifaceted roles of FLOWERING LOCUS T in plant development. Plant Cell Environ. 35, 1742-1755. doi: 10.1111/j.13653040.2012.02558.x

Reed, J. W., Nagatani, A., Elich, T. D., Fagan, M., and Chory, J. (1994). Phytochrome $\mathrm{A}$ and Phytochrome B have overlapping but distinct functions in arabidopsis development. Plant Physiol. 104, 1139-1149. doi: 10.1104/pp.104.4.1139

Sawa, M., Nusinow, D. A., Kay, S. A., and Imaizumi, T. (2007). FKF1 and GIGANTEA complex formation is required for day-length measurement in Arabidopsis. Science 318, 261-265. doi: 10.1126/science.1146994

Shulaev, V., Sargent, D. J., Crowhurst, R. N., Mockler, T. C., Folkerts O., Delcher, A. L., et al. (2011). The genome of woodland strawberry (Fragaria vesca). Nat. Genet. 43, 109-116. doi: 10.1038/ng.740.

Song, Y. H., Smith, R. W., To, B. J., Millar, A. J., and Imaizumi, T. (2012). FKF1 conveys timing information for CONSTANS stabilization in photoperiodic flowering. Science 336, 1045-1049. doi: 10.1126/science.1219644

Sønsteby, A., and Heide, O. M. (2007). Long-day control of flowering in everbearing strawberries. J. Hort. Sci. Biotech. 82, 875-884.

Sønsteby, A., and Heide, O. M. (2008). Long-day rather than autonomous control of flowering in the diploid everbearing strawberry Fragaria vesca ssp semperflorens. J. Hort. Sci. Biotech. 83, 360-366.

Stewart, P. J. (2007). Molecular Characterization of Photoperiodic Flowering in Strawberry (Fragaria sp.). Ph.D. thesis, University of Florida.

Strasser, B., Sánchez-Lamas, M., Yanovsky, M. J., Casal, J. J., and Cerdán, P. D. (2010). Arabidopsis thaliana life without phytochromes. Proc. Natl. Acad. Sci. U.S.A. 107, 4776-4781. doi: 10.1073/pnas.0910446107

Suarez-Lopez, P., Wheatley, K., Robson, F., Onouchi, H., Valverde, F., and Coupland, G. (2001). CONSTANS mediates between the circadian clock and the control of flowering in Arabidopsis. Nature 410, 1116-1120. doi: $10.1038 / 35074138$

Takano, M., Inagaki, N., Xie, X., Kiyota, S., Baba-Kasai, A., Tanabata, T., et al. (2009). Phytochromes are the sole photoreceptors for perceiving red/farred light in rice. Proc. Natl. Acad. Sci. U.S.A. 106, 14705-14710. doi: 10.1073/pnas.0907378106

Tamaki, S., Matsuo, S., Wong, H. L., Yokoi, S., and Shimamoto, K. (2007). Hd3a protein is a mobile flowering signal in rice. Science 316, 1033-1036. doi: $10.1126 /$ science. 1141753

Taoka, K., Ohki, I., Tsuji, H., Furuita, K., Hayashi, K., Yanase, T., et al. (2011). 143-3 proteins act as intracellular receptors for rice Hd3a florigen. Nature 476, 332-335. doi: 10.1038/nature10272
Torti, S., Fornara, F., Vincent, C., Andrés, F., Nordström, K., Göbel, U., et al. (2012). Analysis of the Arabidopsis shoot meristem transcriptome during floral transition identifies distinct regulatory patterns and a leucine-rich repeat protein that promotes flowering. Plant Cell 24, 444-462. doi: 10.1105/tpc.111. 092791

Tsuji, H., Taoka, K., and Shimamoto, K. (2011). Regulation of flowering in rice: two florigen genes, a complex gene network, and natural variation. Curr. Opin. Plant Biol. 14, 45-52. doi: 10.1016/j.pbi.2010.08.016

Valverde, F., Mouradov, A., Soppe, W., Ravenscroft, D., Samach, A., and Coupland, G. (2004). Photoreceptor regulation of CONSTANS protein in photoperiodic flowering. Science 303, 1003-1006. doi: 10.1126/science.1091761

Vince-Prue, D., and Guttridge, C. G. (1973). Floral initiation in strawberryspectral evidence for regulation of flowering by long-day inhibition. Planta 110 , $165-172$.

Wigge, P. A., Kim, M. C., Jaeger, K. E., Busch, W., Schmid, M., Lohmann, J. U., et al. (2005). Integration of spatial and temporal information during floral induction in Arabidopsis. Science 309, 1056-1059. doi: 10.1126/science.1114358

Wollenberg, A. C., Strasser, B., Cerdan, P. D., and Amasino, R. M. (2008). Acceleration of flowering during shade avoidance in Arabidopsis alters the balance between FLOWERING LOCUS C-mediated repression and photoperiodic induction of flowering. Plant Physiol. 148, 1681-1694. doi: 10.1104/pp.108.125468

Yanovsky, M. J., and Kay, S. A. (2002). Molecular basis of seasonal time measurement in Arabidopsis. Nature 419, 308-312. doi: 10.1038/nature00996

Yoo, S. K., Chung, K. S., Kim, J., Lee, J. H., Hong, S. M., Yoo, S. J., et al. (2005). CONSTANS activates SUPPRESSOR OF OVEREXPRESSION OF CONSTANS 1 through FLOWERING LOCUS T to promote flowering in Arabidopsis. Plant Physiol. 139, 770-778. doi: 10.1104/pp.105.066928

Conflict of Interest Statement: The authors declare that the research was conducted in the absence of any commercial or financial relationships that could be construed as a potential conflict of interest.

Received: 31 March 2014; paper pending published: 23 April 2014; accepted: 25 May 2014; published online: 11 June 2014.

Citation: Rantanen M, Kurokura T, Mouhu K, Pinho P, Tetri E, Halonen L, Palonen P, Elomaa $P$ and Hytönen T (2014) Light quality regulates flowering in FvFT1/FvTFL1 dependent manner in the woodland strawberry Fragaria vesca. Front. Plant Sci. 5:271. doi: $10.3389 / \mathrm{fpls} .2014 .00271$

This article was submitted to Plant Genetics and Genomics, a section of the journal Frontiers in Plant Science.

Copyright (C) 2014 Rantanen, Kurokura, Mouhu, Pinho, Tetri, Halonen, Palonen, Elomaa and Hytönen. This is an open-access article distributed under the terms of the Creative Commons Attribution License (CC BY). The use, distribution or reproduction in other forums is permitted, provided the original author(s) or licensor are credited and that the original publication in this journal is cited, in accordance with accepted academic practice. No use, distribution or reproduction is permitted which does not comply with these terms. 